Mathematical Modelling and Analysis

Volume 20 Number 2, March 2015, 148-167

http://dx.doi.org/10.3846/13926292.2015.1019944

(c) Vilnius Gediminas Technical University, 2015
Publisher: Taylor\&Francis and VGTU

http://www.tandfonline.com/TMMA

ISSN: $1392-6292$

eISSN: $1648-3510$

\title{
Singular Orbits and Dynamics at Infinity of a Conjugate Lorenz-Like System
}

\author{
Fengjie Geng ${ }^{a}$ and Xianyi $\mathrm{Li}^{b}$ \\ ${ }^{a}$ School of Science, China University of Geosciences (Beijing) \\ 100083 Beijing, China \\ ${ }^{b}$ College of Mathematical Science, Yangzhou University \\ 225002 Yangzhou, China \\ E-mail(corresp.): mathxyli@yzu.edu.cn \\ E-mail: gengfengjie_hbu@163.com
}

Received March 27, 2014; revised January 7, 2015; published online March 15, 2015

\begin{abstract}
A conjugate Lorenz-like system which includes only two quadratic nonlinearities is proposed in this paper. Some basic properties of this system, such as the distribution of its equilibria and their stabilities, the Lyapunov exponents, the bifurcations are investigated by some numerical and theoretical analysis. The forming mechanisms of compound structures of its new chaotic attractors obtained by merging together two simple attractors after performing one mirror operation are also presented. Furthermore, some of its other complex dynamical behaviours, which include the existence of singularly degenerate heteroclinic cycles, the existence of homoclinic and heteroclinic orbits and the dynamics at infinity, etc, are formulated in detail. In the meantime, some problems deserving further investigations are presented.
\end{abstract}

Keywords: Hopf bifurcation, singularly degenerate heteroclinic cycle, homoclinic and heteroclinic orbits, Poincaré compactification.

AMS Subject Classification: 34C23; 34C37; 34D08; 34D20.

\section{Introduction}

There has been an increasing interest in exploiting chaotic systems (such as the known Chen system [1], Rössler system [16], and Lü systems [13], etc.) since E.N. Lorenz found the first chaotic attractor [12] in a three-dimensional autonomous system. In [7], the authors proposed a new three-dimensional chaotic system [7]

$$
\left\{\begin{array}{l}
\dot{x}=a(y-x), \\
\dot{y}=d y-x z, \\
\dot{z}=-b z+f x^{2}+g x y,
\end{array}\right.
$$

where $a>0, f, g \geq 0, f+g>0, b, d \in \mathbb{R}$, and discussed the stability character of the equilibria, the bifurcations and the existence of homoclinic or heteroclinic 
orbits etc. In 2006, Yang et al. presented a new system called conjugate Lü system [19] as follows

$$
\left\{\begin{array}{l}
\dot{x}=a_{1} x-a_{2} y, \\
\dot{y}=-c y+x z \\
\dot{z}=-b z+x y
\end{array}\right.
$$

where $a_{1}, a_{2}, b, c$ are positive real parameters, and obtained a conjugate Lü attractor in system (1.2) when $\left(a_{1}, a_{2}, c, b\right)=(20,20,36,3)$. Kokubu and Roussarie [5] established the existence of a singularly degenerate heteroclinic cycle in the Lorenz system and some of its other dynamical consequences. For the work about other chaotic systems, see [2,3,4,8,9,10,15,17, 18, 20].

Generally speaking, it is more interesting that a system possesses fewer nonlinear terms and lower dimensional number whereas it can generate chaos. For 3D systems generating chaos, most of them are of type with seven terms, for example, the Lorenz system, the Chen system etc, mentioned above. Based on exploiting chaotic systems and the above system (1.2), we introduce in this paper the following 3D Lorenz-type system with six terms

$$
\left\{\begin{array}{l}
\dot{x}=a(x-y), \\
\dot{y}=-b y+x z, \\
\dot{z}=-c z+x^{2},
\end{array}\right.
$$

where $a>0, b, c \in \mathbb{R}$. Obviously, system (1.3) is not topologically equivalent to system (1.1) or conjugate Lü system. In the sequel we demonstrate that system (1.3) is a chaotic system in the sense of its having a chaotic attractor, a positive largest Lyapunov exponent, a continuous broad frequency spectrum, Poincaré mapping, and the sensitive dependence to initial conditions. See Figure 1.

Initially, by virtue of some numerical simulations, we find some complicate and interesting singular orbits in system (1.3), such as singularly degenerate heteroclinic cycles, homoclinic and heteroclinic orbits etc. These properties with such singular orbits of (1.3), to the best of our knowledge, have not been found in any known literature. This motivates us to further study theoretically and numerically its dynamics, mainly for its singular orbits and dynamics at infinity. Nevertheless, really, we don't know the physical background of system (1.3) at least at present.

The rest of the paper is organized as follows. The local behaviours of system (1.3), such as the distribution of equilibria and their stabilities, the bifurcations and the forming mechanism of its compound structure are discussed in Section 2. In Section 3, we establish the existence of singularly degenerate heteroclinic cycles, homoclinic and heteroclinic orbits. Its dynamics at infinity is presented in Section 4. Finally, some conclusions are drawn in Section 5.

\section{Local Behaviour of System (1.3)}

In this section divided into six subsections, we discuss the local behaviour of system (1.3). 


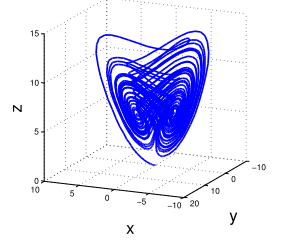

(a)

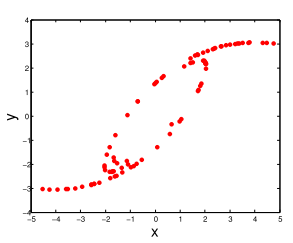

(d)

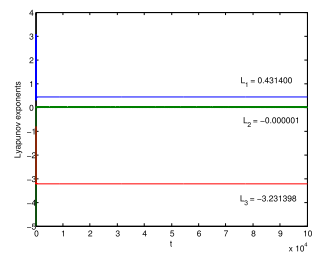

(b)

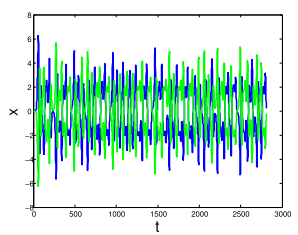

(e)

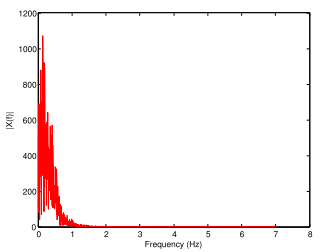

(c)

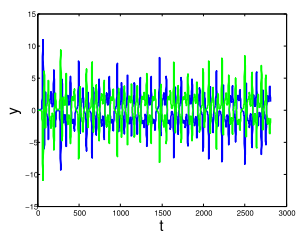

(f)

Figure 1. (a) Chaotic attractor of system (1.3), (b) its Lyapunov exponents $L_{1}=0.4314$, $L_{2}=-0.000001$ and $L_{3}=-3.231398$, (c) single-sided amplitude spectrum of $|x(t)|$, (d) the Poincaré map of $x-y$ plane for $z=6$, (e) time series of $x(t)$ for $\left(x_{0}, y_{0}, z_{0}\right)=( \pm 3.82 e-5$, $\pm 6.18 e-5,0)$, (f) time series of $y(t)$ for $\left(x_{0}, y_{0}, z_{0}\right)=( \pm 3.82 e-5, \pm 6.18 e-5,0)$ of system (1.3) for the parameters $(a, b, c)=(3,5,0.8)$. These figures display that system (1.3) is a chaotic one.

\subsection{Distribution of equilibrium of system (1.3)}

By a simple analysis, one may derive the following consequence.

Theorem 1. The distribution of equilibrium of system (1.3) is in detail described in Table 1 when the parameters $a>0, b$, c are varying in $\mathbb{R}^{2}$.

Table 1. The distribution of equilibrium of system (1.3).

\begin{tabular}{lll}
\hline$b$ & $c$ & distribution of equilibrium \\
\hline & $=0$ & non-isolated $E_{z}=(0,0, z)$ \\
$b c \leq 0$ & $\neq 0$ & unique $E_{0}=(0,0,0)$ \\
$b c>0$ & $\neq 0$ & $E_{0}=(0,0,0)$ and $E_{ \pm}=( \pm \sqrt{b c}, \pm \sqrt{b c}, b)$ \\
\hline
\end{tabular}

Remark 1 . We may easily see from Table 1 that there is an occurrence of pitchfork bifurcation for system (1.3) when $a, b, c$ lie in the space $\left\{(a, b, c) \in \mathbb{R}^{+} \times \mathbb{R}^{2} \mid\right.$ $b c=0\}$.

\subsection{Behaviour of equilibrium $E_{0}$}

According to the linearized analysis and the center manifold theory, one can achieve the following result.

Theorem 2. The behaviour of equilibrium $E_{0}$ of system (1.3) is totally summarized in Table 2 for $b, c \in \mathbb{R}^{2}$. 
Table 2. The behaviour of equilibrium $E_{0}$ of system (1.3).

\begin{tabular}{|c|c|c|c|}
\hline$b$ & $c$ & Type of $E_{0}$ & Property of $E_{0}$ \\
\hline$<0$ & $\begin{array}{l}<0 \\
=0 \\
>0\end{array}$ & $\begin{array}{l}\text { node } \\
\text { non-hyperbolic } \\
\text { saddle-node }\end{array}$ & $\begin{array}{l}\text { a } 3 \mathrm{D} W_{l o c}^{u} \\
\text { a } 1 \mathrm{D} W_{l o c}^{c} \text { and a } 2 \mathrm{D} W_{l o c}^{u} \\
\text { a } 1 \mathrm{D} W_{l o c}^{s} \text { and a } 2 \mathrm{D} W_{l o c}^{u}\end{array}$ \\
\hline$=0$ & $\begin{array}{l}<0 \\
=0 \\
>0\end{array}$ & $\begin{array}{l}\text { non-hyperbolic } \\
\text { non-hyperbolic } \\
\text { non-hyperbolic }\end{array}$ & $\begin{array}{l}\text { a } 1 \mathrm{D} W_{l o c}^{s} \text { and a } 2 \mathrm{D} W_{l o c}^{u} \\
\text { a } 2 \mathrm{D} W_{l o c}^{c} \text { and a } 1 \mathrm{D} W_{l o c}^{u} \\
\text { a } 1 \mathrm{D} W_{l o c}^{s} \text { and a } 2 \mathrm{D} W_{l o c}^{u}\end{array}$ \\
\hline$>0$ & $\begin{array}{l}<0 \\
=0 \\
>0\end{array}$ & $\begin{array}{l}\text { saddle-node } \\
\text { non-hyperbolic } \\
\text { saddle-node }\end{array}$ & $\begin{array}{l}\text { a } 1 \mathrm{D} W_{l o c}^{s} \text { and a } 2 \mathrm{D} W_{l o c}^{u} \\
\text { a } 1 \mathrm{D} W_{l o c}^{s} \text {, a } 1 \mathrm{D} W_{l o c}^{c} \text { and a } 2 \mathrm{D} W_{l o c}^{u} \\
\text { a } 2 \mathrm{D} W_{l o c}^{s} \text { and a } 1 \mathrm{D} W_{l o c}^{u}\end{array}$ \\
\hline
\end{tabular}

Remark 2. For $b=0$, the results of equilibrium $E_{0}$ in Table 2 are obtained by Center Manifold Theorem.

\subsection{Behavior of equilibrium $E_{z}$}

If $c=0$, then system (1.3) has non-isolated equilibria $E_{z}=(0,0, z)$ for any $z \in$ $\mathbb{R}$ with three eigenvalues $\lambda_{1,2}=\frac{(a-b) \pm \sqrt{(a-b)^{2}+4(a b-a z)}}{2}$ and $\lambda_{3}=0$. Therefore, we may easily derive the following results.

Theorem 3. Assume that $c=0$, then system (1.3) has non-isolated equilibria $E_{z}=(0,0, z)$ for any $z \in \mathbb{R}$. Moreover, the local dynamical behaviours of any one are formulated in Table 3.

Table 3. The property of equilibrium $E_{z}$ of system (1.3).

\begin{tabular}{lll}
\hline$a-b$ & $z$ & Property of $E_{z}$ \\
\hline \multirow{3}{*}{$<0$} & $b<z$ & a $2 \mathrm{D} W_{l o c}^{s}$ and a $1 \mathrm{D} W_{l o c}^{c}$ \\
& $b=z$ & a $1 \mathrm{D} W_{l o c}^{s}$ and a $2 \mathrm{D} W_{l o c}^{c}$ \\
& $b>z$ & a $1 \mathrm{D} W_{l o c}^{s}$, a $1 \mathrm{D} W_{l o c}^{c}$ and a $1 \mathrm{D} W_{l o c}^{u}$ \\
\hline \multirow{3}{*}{0} & $b<z$ & fold-Hopf bifurcation occurs \\
& $b=z$ & a $3 \mathrm{D} W_{l o c}^{c}$ \\
& $b>z$ & a $1 \mathrm{D} W_{l o c}^{s}$, a $1 \mathrm{D} W_{l o c}^{c}$ and a $1 \mathrm{D} W_{l o c}^{u}$ \\
\hline & $b<z$ & a $1 \mathrm{D} W_{l o c}^{c}$ and a $2 \mathrm{D} W_{l o c}^{u}$ \\
& $b=z$ & a $2 \mathrm{D} W_{l o c}^{c}$ and a $1 \mathrm{D} W_{l o c}^{u}$ \\
& $b>z$ & a $1 \mathrm{D} W_{l o c}^{s}$, a $1 \mathrm{D} W_{l o c}^{c}$ and a $1 \mathrm{D} W_{l o c}^{u}$ \\
\hline
\end{tabular}

\subsection{Behavior of equilibria $E_{ \pm}$}

According to the symmetry of $E_{+}$and $E_{-}$in system (1.3), it is sufficient to study the behaviour of equilibrium $E_{+}$. The characteristic equation associated with the equilibrium $E_{+}$is

$$
\lambda^{3}-(a-b-c) \lambda^{2}-c(a-b) \lambda+2 a b c=0 .
$$


Notice that $a, b$ and $c$ belong to the set $W=\left\{(a, b, c) \in \mathbb{R}^{+} \times \mathbb{R}^{2} \mid a>0\right.$, $b c>0\}$. For convenience of discussion in the sequel, we define the sets $W_{1}, W_{2}$ and $W_{3}$ as follows:

$$
\begin{aligned}
& W_{1}=\{(a, b, c) \in W: b>0, c>0, a \geq b\}, \\
& W_{2}=\{(a, b, c) \in W: b>0, c>0, a<b\}, \\
& W_{3}=\{(a, b, c) \in W: b<0, c<0\} .
\end{aligned}
$$

Obviously, $W=W_{1} \cup W_{2} \cup W_{3}$. Further define the subsets $W_{21}, W_{22}, W_{23}, W_{24}$ of $W_{2}$ as

$$
\begin{aligned}
& W_{21}=\left\{(a, b, c) \in W_{2}: c<\frac{(a-b)^{2}-2 a b}{a-b}\right\}, \\
& W_{22}=\left\{(a, b, c) \in W_{2}: c=\frac{(a-b)^{2}-2 a b}{a-b} \triangleq c_{0}\right\}, \\
& W_{23}=\left\{(a, b, c) \in W_{2}: c>\frac{(a-b)^{2}-2 a b}{a-b}>0\right\}, \\
& W_{24}=\left\{(a, b, c) \in W_{2}: \frac{(a-b)^{2}-2 a b}{a-b} \leq 0\right\} .
\end{aligned}
$$

By applying the Routh-Hurwitz criterion and some analytical computations, it is easy to get the following results.

Theorem 4. The equilibrium $E_{+}$of system (1.3) is unstable for $(a, b, c) \in$ $W_{1} \cup W_{21} \cup W_{3}$ whereas asymptotically stable for $(a, b, c) \in W_{23} \cup W_{24}$.

\subsection{Hopf bifurcation of equilibria $E_{ \pm}$}

From the above Theorem 4, we can see that there will be an occurrence of bifurcation for $(a, b, c) \in W_{22}$. Then, what kind of bifurcation is it? How about its stability? In this subsection, we will answer these questions. Firstly, we introduce the following lemmas which will be significant for Theorem 5 .

Lemma 1. When $(a, b, c) \in W_{22}$ and choose the parameter $c$ as a bifurcation parameter, system (1.3) undergoes a Pioncaré-Andronov-Hopf bifurcation bifurcation (or simply a Hopf bifurcation) at $E_{+}$.

Proof. For $(a, b, c) \in W_{22}$, it follows that Eq. (2.1) has one negative real root $\lambda_{1}=\frac{2 a b}{a-b}$ and a pair of conjugate purely imaginary roots $\lambda_{2,3}= \pm \omega_{0} i$ with $\omega_{0}=\sqrt{2 a b-(a-b)^{2}}$. Taking into account that $\operatorname{Re}\left(\lambda_{2}\right)=0$ at $c=c_{0}$, we obtains

$$
\left.\frac{d R e\left(\lambda_{2}\right)}{d c}\right|_{c=c_{0}}=-\frac{\left[2 a b-(a-b)^{2}\right](a-b)^{2}}{2\left[2 a b-(a-b)^{2}\right](a-b)^{2}+8 a^{2} b^{2}}<0,
$$

which means for $(a, b, c) \in W_{22}$, the transversal condition always holds. Also, $\operatorname{Re}\left(\lambda_{1}\right)=\lambda_{1}<0$. Therefore, all conditions for Hopf bifurcation [6] to occur are fulfilled. Consequently, a Hopf bifurcation happens at $E_{+}$. 
The proof for this lemma is then finished.

Next, we will study the stability of the periodic orbit bifurcated from $E_{+}$ for the parameters in $W_{22}$ by using the Projection Method in [6]. The following proposition may be derived.

Lemma 2. For $(a, b, c) \in W_{22}$, the first Lyapunov coefficient of system (1.3) at $E_{+}$is given by

$$
l_{1}(a, b)=-\frac{a^{2}(a-b)^{2}\left[(a-b)^{2}-2 a b\right] N_{1}}{b D_{1} D_{2}},
$$

where

$$
\begin{aligned}
& N_{1}=\left[3(a-b)^{2}+a b\right]\left[(a-b)^{2}-2 a b\right]+a^{2} b^{2}, \\
& D_{1}=(a-b)^{2}\left[(a-b)^{2}-2 a b\right]-4 a^{2} b^{2}<0, \\
& D_{2}=(a-b)^{2}\left[(a-b)^{2}-2 a b\right]-a^{2} b^{2}<0 .
\end{aligned}
$$

Proof. When $(a, b, c) \in W_{22}, c=c_{0}$, and $E_{+}=\left(\sqrt{b c_{0}}, \sqrt{b c_{0}}, b\right)$. Take the change of the variables $x_{1}=x-\sqrt{b c_{0}}, \quad x_{2}=y-\sqrt{b c_{0}}$ and $x_{3}=z-b$, which transforms $E_{+}$to $E_{0}$ and system (1.3) into

$$
\left\{\begin{array}{l}
\dot{x_{1}}=a\left(x_{1}-x_{2}\right) \\
\dot{x_{2}}=b x_{1}-b x_{2}+\sqrt{b c_{0}} x_{3}+x_{1} x_{3}, \\
\dot{x_{3}}=2 \sqrt{b c_{0}} x_{1}-c_{0} x_{3}+x_{1}^{2} .
\end{array}\right.
$$

Denoting $u=\sqrt{\frac{b\left[(a-b)^{2}-2 a b\right]}{a-b}}$, we have

$$
A=\left(\begin{array}{ccc}
a & -a & 0 \\
b & -b & u \\
2 u & 0 & -c_{0}
\end{array}\right)
$$

It is easy to derive that $A$ has a real eigenvalue $\lambda_{1}=a-b-c_{0}$ and a pair of purely imaginary eigenvalues $\lambda_{2,3}= \pm \omega_{0} i$.

With some tedious calculations we show that

$$
p=\frac{1}{H}\left(\begin{array}{c}
-b-\omega_{0} i \\
-a \\
-\frac{a u}{-\omega_{0} i+c_{0}}
\end{array}\right) \quad \text { and } \quad q=\left(\begin{array}{c}
-a \\
-a+\omega_{0} i \\
-\frac{2 a u}{\omega_{0} i+c_{0}}
\end{array}\right)
$$

satisfy $A q=i \omega_{0} q, A^{T} p=-i \omega_{0} p,\langle p, q\rangle=\sum_{i=1}^{3} \bar{p}_{i} q_{i}=1$, where

$$
H=-a b+2 a \omega_{0} i+a^{2}-\frac{2 a^{2} u^{2}}{-c_{0}^{2}+2 c_{0} \omega_{0} i+\omega_{0}^{2}} .
$$


Some further computations yield to $h_{11}=\left(h_{11}^{1}, h_{11}^{2}, h_{11}^{3}\right)$, where

$$
\left\{\begin{array}{l}
h_{11}^{1}=-\frac{a^{2}}{u}-\frac{2 a^{2} c_{0}^{2}}{u\left(c_{0}^{2}+\omega_{0}^{2}\right)} \\
h_{11}^{2}=-\frac{a^{2}}{u}-\frac{2 a^{2} c_{0}^{2}}{u\left(c_{0}^{2}+\omega_{0}^{2}\right)} \\
h_{11}^{3}=-\frac{4 a^{2} c_{0}}{c_{0}^{2}+\omega_{0}^{2}}
\end{array}\right.
$$

and $h_{20}=\left(h_{20}^{1}, h_{20}^{2}, h_{20}^{3}\right)$, where

$$
\left\{\begin{array}{l}
h_{20}^{1}=\frac{a^{3} u}{K_{4}}+\frac{2 a^{3} u K_{1}}{K_{3}} \\
h_{20}^{2}=\frac{a^{2} u K_{2}}{K_{4}}+\frac{2 a^{2} u K_{2} K_{1}}{K_{3}}, \\
h_{20}^{3}=\frac{2 a^{2}\left(-b \omega_{0} i+2 \omega_{0}^{2}+a \omega_{0} i\right)}{K_{4}}-\frac{4 a^{3} u^{2}}{K_{3}}
\end{array}\right.
$$

with $K_{1}=c_{0}+2 \omega_{0} i, K_{2}=a-2 \omega_{0} i, K_{3}=\left(c_{0}+\omega_{0} i\right)$ and $K_{4}=-2 a \omega_{0}^{2}-a u^{2}+$ $2 b \omega_{0}^{2}+2 c_{0} \omega_{0}^{2}+\left(4 \omega_{0}^{3}+a c_{0}-b c_{0}\right) \omega_{0} i$.

After further performing some calculations as described in [6], we may derive the first Lyapunov coefficient of system (1.3) at $E_{+}$which is just expressed by (2.2).

Now define the function $f(x)=3 x^{4}-17 x^{3}+27 x^{2}-17 x+3$ with $x \in$ $(0,1)$. It is easy to prove that $f^{\prime}(x)=12 x^{3}-51 x^{2}+54 x-17<0$ for $x \in(0,1)$ because $12 x^{3}-51 x^{2}+54 x-17=0$ has a unique real root $x=$ $\frac{17+(663+9 \sqrt{46})^{1 / 3}-(663-9 \sqrt{46})^{1 / 3}}{12}>1$. Therefore, the function $f(x)=3 x^{4}-$ $17 x^{3}+27 x^{2}-17 x+3$ has a unique real root $x_{0} \in(0,1)$ because of $f(0)>0$ and $f(1)<0$.

Notice that $l_{1}(a, b)>(=,<) 0 \Leftrightarrow N_{1}>(=,<) 0 \Leftrightarrow$ the function $f(x)=$ $3 x^{4}-17 x^{3}+27 x^{2}-17 x+3>(=,<) 0$ for $x \in(0,1) \Leftrightarrow 0<x<(=,>) x_{0}$.

Hence, summarizing the above discussions we finally obtain the main result of this section as follows.

Theorem 5. System (1.3) undergoes a Pioncaré-Andronov-Hopf bifurcation at $E_{+}$for $(a, b, c) \in W_{22}$. More precisely, for $b>a>x_{0} b$, where $x_{0}$ is the unique positive root of $f(x)=3 x^{4}-17 x^{3}+27 x^{2}-17 x+3$ for $x \in(0,1)$, the Hopf bifurcation is stable; for $0<a<x_{0} b$, the Hopf bifurcation is unstable. Namely, for each $c>c_{0}$, but close to $c_{0}$, there exists an unstable closed orbit near the asymptotically stable $E_{+}$; for $a=x_{0} b$, the Hopf bifurcation is degenerate, and the second or the third or even more higher order Lyapunov coefficients needs computing to determine the stability of the bifurcated periodic orbit. Symmetrically, there are the same results at $E_{-}$for $(a, b, c) \in W_{22}$.

\subsection{Forming mechanism for the structure of new chaotic attractor}

In order to reveal the forming mechanism for the structure of this new chaotic attractor, the controlled system of system (1.3) is proposed, namely, the fol- 


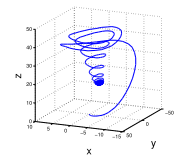

(a) $d=36$

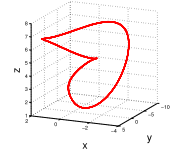

(f) $d=0.4$

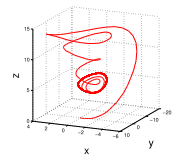

(b) $d=3$

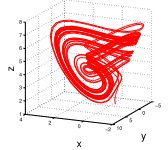

(g) $d=-0.4$

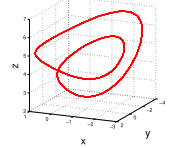

(c) $d=1.2$

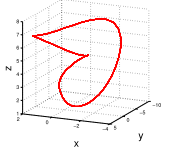

(h)

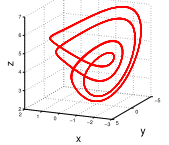

(d) $d=0.8$

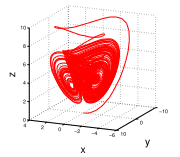

(i) $d=0.2$

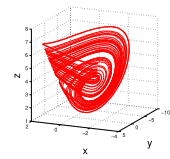

(e) $d=0.5$

Figure 2. The phase portraits of system (2.3) when the parameters $(a, b, c)=(2,3,0.8)$ and $d$ varies and the initial condition $\left(x_{0}, y_{0}, z_{0}\right)=(3.82 \times 1 e-5,6.18 \times 1 e-5,0)$. These figures display the evolution process of chaotic attractors of system (1.3).

lowing system of autonomous differential equations

$$
\left\{\begin{array}{l}
\dot{x}=a(x-y) \\
\dot{y}=-b y+x z+d, \\
\dot{z}=-c z+x^{2}
\end{array}\right.
$$

In this system, $d$ is a parameter of control with its value varying in $\mathbb{R}$. When the parameter $d$ is changed, the chaos behaviour of system (1.3) can be effectively controlled. So, it is a controller. Here, we allow the initial values of the system to be selected as $\left(x_{0}, y_{0}, z_{0}=(3.82 \times 1 e-5,6.18 \times 1 e-5,0)\right.$. One can observe different dynamical behavior of system (2.3) at different parameter $d$. Different dynamical behaviour can be summarized as follows (see Figure 2).

(a) When $|d| \geq 35$, system (1.3) converges to a point.

(b) When $1.5<|d|<35$, system (1.3) has limit cycles.

(c) When $0.93<|d| \leq 1.5$, system (1.3) demonstrates period-doubling bifurcations.

(d) When $0.63<|d| \leq 0.93$, system (1.3) demonstrates Multi-Cycle dynamics.

(e) When $0.41 \leq|d| \leq 0.63$, system (1.3) becomes a left (or a right) halfimage attractor.

(f) When $d=0.4$, system (1.3) displays a limit cycle, while it is a left half-image attractor for $d=-0.4$.

(g) When $0.39 \leq|d|<0.40$, system (1.3) displays a limit cycle.

(h) When $0.13 \leq|d|<0.39$, system (1.3) demonstrates partial attractors, which are bounded.

(i) When $|d|<0.13$, system (1.3) exhibits a complete attractor.

In the controller, one can see, when $|d|$ is large enough, chaos attractor disappears; when $|d|$ is small enough, a complete chaos attractor appears. So, $|d|$ is an important parameter to control chaos in the nonlinear-system.

This means that, the transverse butterfly attractor reported is also a compound structure obtained by merging together two simple attractors after per- 
forming one mirror operation.

\section{Existence of Singular Orbits and Some Conjectures for System (1.3)}

In this section, we study other complicate dynamical behaviours of system (1.3), mainly for the existence of singular orbits, such as singularly degenerate heteroclinic cycle, homoclinic and heteroclinic orbit, etc, which play some key roles in revealing the fascinating nature of system (1.3).

\subsection{Singularly degenerate heteroclinic cycle}

Recall that a singularly degenerate heteroclinic cycle consists of an invariant set formed by a line of equilibria together with a heteroclinic orbit connecting two of the equilibria. Kokubu and Roussarie [5] began to study the existence of such cycle in Lorenz system by classical method "blow-up of the vector field at infinity". In 2009, Messias [15] discovered a set of infinitely many such kind of cycles from Lorenz system combining analytical and numerical techniques. Other Lorenz-like systems $[2,11,17,20]$ have such kind of properties, too. In 2012, Liu [10] found that a conjugate Lorenz-type system has infinitely many such kind of cycles. So, we naturally propose the problem: Does system (1.3) possess such properties?

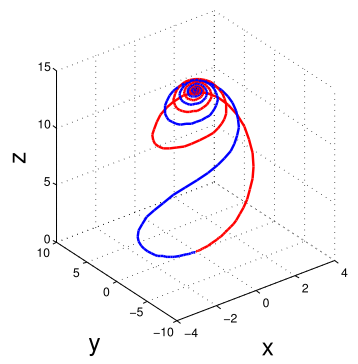

(a)

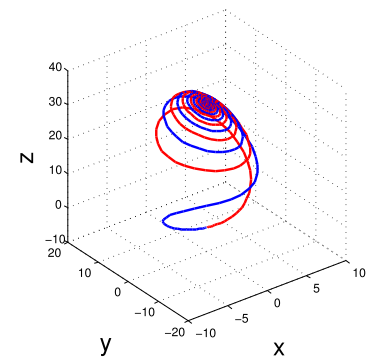

(b)

Figure 3. Two orbits of system (1.3) for the parameters $a=2, b=3$ and $c=0$, time integration: $[0,250]$ and the initial values: (a) $\left(x_{0}, y_{0}, z_{0}\right)=( \pm 3.82 \times 1 e-5, \pm 6.18 \times 1 e-5,0),(\mathrm{b})$

$\left(x_{0}, y_{0}, z_{0}\right)=( \pm 3.82 \times 1 e-5, \pm 6.18 \times 1 e-5,-3)$. These figures show the existence of two singularly degenerate heteroclinic cycles of system (1.3).

By numerical simulations, it has been found that system (1.3) has this kind of singular cycles, see Figure 3. Precisely, one has the numerical results as follows.

Numerical Result 3.1. For $c=0$ and $0<a<b$, the 1D unstable manifold $W^{u}\left(E_{1}\right)$ of each normally hyperbolic saddle-like $P_{1}=\left(0,0, z_{1}\right)\left(z_{1} \in(-\infty, b)\right)$ of system (1.3) tends to one of the normal hyperbolic stable foci-like $P_{2}=$ $\left(0,0, z_{2}\right)\left(z_{2} \in(b,+\infty)\right)$ discussed in Theorem 3 , forming singularly degenerate 


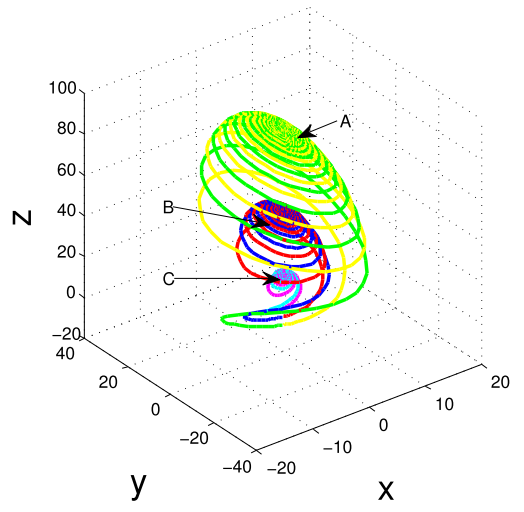

Figure 4. Singularly degenerate heteroclinic cycles of system (1.3) with (A) $\left(x_{0}, y_{0}, z_{0}\right)=( \pm 3.82 \times 1 e-5, \pm 6.18 \times 1 e-5,-7),(\mathrm{B})$ $\left(x_{0}, y_{0}, z_{0}\right)=( \pm 3.82 \times 1 e-5, \pm 6.18 \times 1 e-5,-3)$ and $(\mathrm{C})$

$\left(x_{0}, y_{0}, z_{0}\right)=( \pm 3.82 \times 1 e-5, \pm 6.18 \times 1 e-5,1)$ when $(a, b, c)=(3,4,0)$. These figures suggest that system (1.3) has infinitely many singularly degenerate heteroclinic cycles.

heteroclinic cycles (see Figure 3-Figure 4). This also implies that system (1.3) has an infinite set of singularly degenerate heteroclinic cycles, too.

The above results have not been theoretically proved for the existence of degenerate heteroclinic cycles. Hence, with the similar method used in [5], we take the linear transformation $u=\sqrt{a} x, v=a \sqrt{a}(x-y), w=a z$ that converts system (1.3) into the following system

$$
\left\{\begin{array}{l}
\dot{u}=v \\
\dot{v}=a b u-(b-a) v-u w, \\
\dot{w}=-c w+u^{2}
\end{array}\right.
$$

It is easy to see that system (3.1) with $c=0$ and $b>a>0$ (i.e. system (1.3)) is similar to Shimizu-Morioka system

$$
\left\{\begin{array}{l}
\dot{x}=y, \\
\dot{y}=A x-y-x z, \\
\dot{z}=-c z+x^{2},
\end{array}\right.
$$

where $A>0$ and $c=0$, and it is a special case of Lorenz system

$$
\left\{\begin{array}{l}
\dot{x}=y, \\
\dot{y}=A x-B y-x z-x^{3}, \\
\dot{z}=-\beta z+x^{2}
\end{array}\right.
$$

with $A, B>0, \beta=0$ on pp.493 of [14] or pp. 521-522 of [5] whose cubic term $x^{3}$ is removed. According to [5] and [3], there exists a singularly degenerate heteroclinic cycle in Shimizu-Morioka system and Lorenz system respectively. By using the similar reasoning in [5] and [3], one sees that system (1.3) has this property, too. However, up to now, we can not give a rigorous theoretical proof for the existence of infinitely many singularly degenerate heteroclinic cycles of system (1.3). So a conjecture is presented as follows. 
Conjecture 1. Consider system (1.3) with $c=0$. Then, for $b>a>0$, system (1.3) has a set of infinitely many singularly degenerate heteroclinic cycles.

When $c$ passes through zero, we find new chaotic attractors with the singularly degenerate heteroclinic cycles disappearing, for example, see Figure 5.

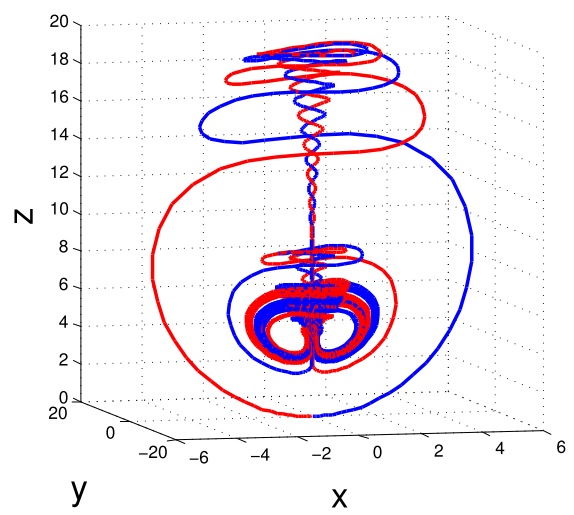

Figure 5. The phase portrait of system (1.3) for $(a, b, c)=(3,4,0.1)$, time of integration: $[0,750]$, and initial conditions: $\left(x_{0}, y_{0}, z_{0}\right)=( \pm 3.82 \times 1 e-5, \pm 6.18 \times 1 e-5,0)$. The figure suggests the existence of chaotic attractors which are bifurcated from the singularly degenerate heteroclinic cycles of system (1.3).

\subsection{Existence of homoclinic and heteroclinic orbits}

In this subsection we study global bifurcations corresponding to the appearance of homoclinic or heteroclinic orbits connecting hyperbolic equilibria in system (1.3). Does system (1.3) have such kind of properties? On the one hand, we consider the existence of homoclinic orbits of system (1.3). When $a=2, b=3$, $c=8.037$ (At this time, the parameters $a, b$ and $c$ satisfy $b>a>0$ and $0<c<\frac{(a-b)^{2}-2 a b}{a-b}$, and $E_{0}$ is a saddle-node, $E_{ \pm}$are saddle-focus according to Section 2.4.) and $\left(x_{0}, y_{0}, z_{0}\right)=( \pm 3.82 \times 1 e-5, \pm 6.18 \times 1 e-5,0)$, numerical simulations show that system (1.3) has two homoclinic orbits to $E_{0}$ ( refer to Figure 6).

Whether or not does system (1.3) have other homoclinic orbits such as to $E_{+}$or $E_{-}$? So far, we can not give theoretical answer. Hence, we propose the second problem as follows.

Conjecture 2. When $b>a>0,2 a b>(a-b)^{2}$, and some $c_{0} \in\left(0, \frac{(a-b)^{2}-2 a b}{a-b}\right)$, system (1.3) has two homoclinic orbits to $E_{+}$or $E_{-}$.

On the other hand, one studies the existence of heteroclinic orbits of system (1.3). First of all, after making a careful analysis of system (1.3) in [7] (or the system (1) in [8]), we find that system (1.1) has two heteroclinic orbits when its equilibrium $S_{0}$ is unstable and $S_{ \pm}$are local asymptotically stable. (Note: $S_{0}$ and $S_{ \pm}$in [7] are corresponding to $E_{0}$ and $E_{ \pm}$in this paper.)

However, one also finds that $E_{0}$ is unstable and $E_{ \pm}$are asymptotically stable when $(a, b, c) \in W_{24}$. Inspired by this idea, we make some computer simulations 


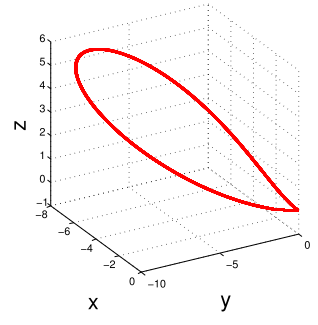

(a)

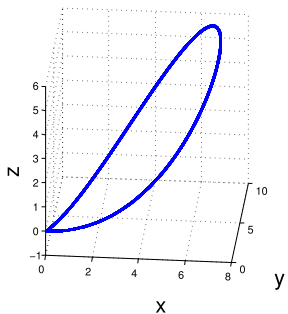

(b)

Figure 6. Two homoclinic orbits of system (1.3) to $E_{0}$ when $a=2, b=3, c=8.037$ and the different initial values $\left(x_{0}, y_{0}, z_{0}\right)$ : (a) $\left(x_{0}, y_{0}, z_{0}\right)=(-3.82 \times 1 e-5,-6.18 \times 1 e-5,0)$, (b) $\left(x_{0}, y_{0}, z_{0}\right)=(3.82 \times 1 e-5,6.18 \times 1 e-5,0)$.

for system (1.3) and discover two heteroclinic orbits of system (1.3) to $E_{0}$ and $E_{ \pm}$respectively. For the numerical results, see Figure 7.

Notice that $E_{0}$ is unstable at the case $(a, b, c) \in W_{23}$ whereas $E_{ \pm}$are asymptotically stable in this case. Now set $a=2, b=3, c=11.1,400$, which obviously satisfy $(a, b, c) \in W_{23}$, and $\left(x_{0}, y_{0}, z_{0}\right)=( \pm 3.82 \times 1 e-5, \pm 6.18 \times 1 e-5,0)$. Numerical simulations illustrate that system (1.3) also has heteroclinic orbits to $E_{0}$ and $E_{ \pm}$respectively (see Figure 8). Whether or not does it have other heteroclinic orbits such as the ones joining the equilibria $E_{+}$and $E_{-}$? So far, we fail to answer this question. Hence, we present the following third conjecture.

Conjecture 3 . Assume $(a, b, c) \in W_{23}$ or $W_{24}$, then system (1.3) has two heteroclinic orbits joining $E_{0}$ and $E_{ \pm}$respectively.

\subsection{Numerical results of other singular orbits}

Set the initial values $\left(x_{0}, y_{0}, z_{0}\right)=(3.82 \times 1 e-5,6.18 \times 1 e-5,0)$ and the parameters $a=2, b=3$ whereas $c$ varies in $\mathbb{R}$. We discover that the portraits of system (1.3) illustrate diverse interesting and fascinating phenomena, refer to Figure 9. The different dynamical behaviour of system (1.3) can be numerically observed as follows.

(a) When $0.76<c \leq 0.812$, system (1.3) demonstrates period-doubling bifurcations.

(b) When $0.812<c \leq 8.036$, system (1.3) displays a limit cycle encircling $E_{ \pm}$and $E_{0}$.

(c) When $8.05<c<11$, system (1.3) shows a limit cycle encircling $E_{+}$ or $E_{-}$.

\section{Dynamical Behaviour of System (1.3) at Infinity}

In this section, we shall make an analysis of the flow of system (1.3) near and at infinity. 


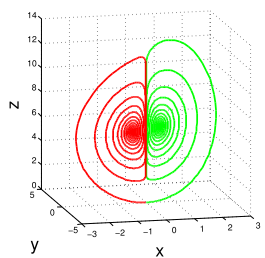

(a) $c=0.05$

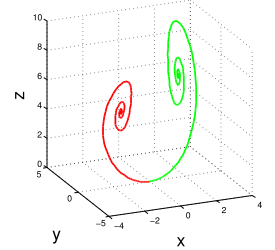

(c) $c=1$

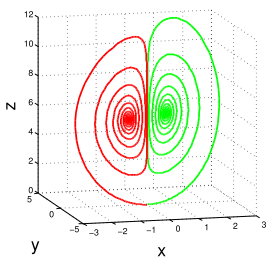

(b) $c=0.1$

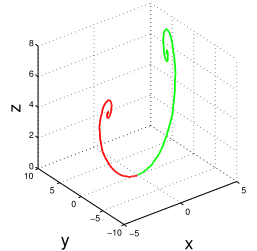

(d) $c=3$

Figure 7. Two orbits of system (1.3) for the parameters $(a, b)=(1,6)$ and $c$ varies satisfying $c>0$, the initial conditions $\left(x_{0}, y_{0}, z_{0}\right)=( \pm 3.82 \times 1 e-5, \pm 6.18 \times 1 e-5,0)$ and time integration: $[0,750]$. These figures display the existence of two heteroclinic orbits of system (1.3) joining $E_{0}$ and $E_{ \pm}$respectively.

\subsection{Statement of main results}

The main results in this subsection are summarized in the following theorems.

Theorem 6. For $(a, b, c) \in \mathbb{R}^{+} \times \mathbb{R}^{2}$, the phase portrait of system (1.3) on the sphere at infinity is as illustrated in Figure 10, and there exists only a circle of equilibria containing the endpoints of the $y$ - and $z$-axis.

Since $c=0$ and $(a, b) \in \mathbb{R}^{+} \times \mathbb{R}$, the dynamics of system (1.3) has been discussed in Section 3, we consider the case for $c \neq 0$ in the next theorem.

Theorem 7. If $c \neq 0$, then system (1.3) has two infinite heteroclinic orbits, one of which consists of the origin $E_{0}$, the positive portion of the $z$-axis and of one equilibrium on the sphere at infinity (the endpoint of the positive $z$-axis); the other consists of $E_{0}$, the negative part of the $z$-axis and of one equilibrium on the sphere at infinity (the endpoint of the negative z-axis). Moreover, the origin is asymptotically stable for $c>0$ along the $z$-axis while unstable for $c<0$ (see Figure 11).

The section is organized as follows: in Subsection 4.2, we study the dynamics of system (1.3) in a neighbourhood of and on the sphere at infinity and consequently to prove Theorem 6 by using Poincaré compactification [15]. Based on the knowledge of the dynamical behaviour near and at infinity, we shall prove Theorem 7 in Subsection 4.3.

\subsection{Dynamical behaviour of system (1.3) near and at infinity}

In this subsection we make an analysis of the flow of system (1.3) near and at infinity. In order to do so, in the following four subsections, we shall analyze 


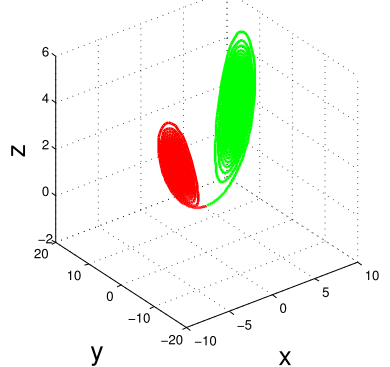

(a) $c=11.1$

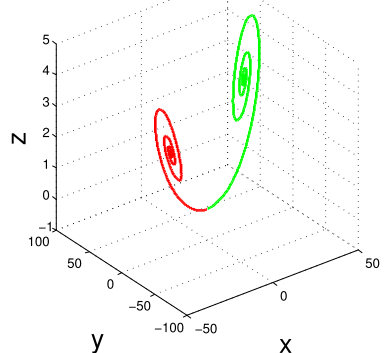

(b) $c=400$

Figure 8. Two heteroclinic orbits of system (1.3) when $\left(x_{0}, y_{0}, z_{0}\right)=( \pm 3.82 \times 1 e-5, \pm 6.18 \times 1 e-5,0),(a, b)=(2,3)$, different values of $c$ and time of integration: $[0,750]$.

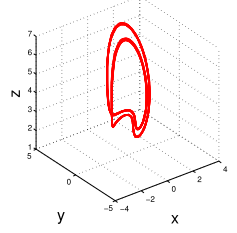

(a) $c=0.78$

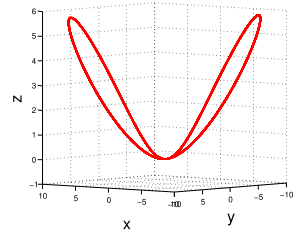

(b) $c=8.036$

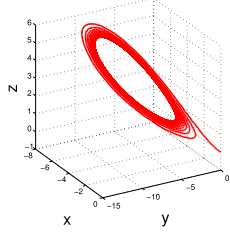

(c) $c=10$

Figure 9. The evolutions of dynamics of system (1.3) when the parameters $a=2, b=3$ and $c$ varies and the initial value $\left(x_{0}, y_{0}, z_{0}\right)=(-3.82 \times 1 e-5,-6.18 \times 1 e-5,0)$ : (a) period-doubling bifurcations, (b) a limit cycle encircling $E_{ \pm}$, (c) a limit cycle encircling $E_{+}$ or $E_{-}$.

the Poincaré compactification of system (1.3) in the local charts $U_{i}$ and $V_{i}$, $i=1,2,3$.

\subsubsection{In the local charts $U_{1}$ and $V_{1}$}

The expression of the Poincaré compactification $p(X)$ of system (1.3) in the local chart $U_{1}$ is given by

$$
\left\{\begin{array}{l}
\dot{z_{1}}=-(a+b) z_{1} z_{3}+a z_{1}^{2} z_{3}+z_{2} \\
\dot{z_{2}}=-(a+c) z_{2} z_{3}+a z_{1} z_{2} z_{3}+1 \\
\dot{z_{3}}=a z_{1} z_{3}^{2}-a z_{3}^{2}
\end{array}\right.
$$

Taking $z_{3}=0$ (which corresponds to the points on the sphere $\mathbb{S}^{2}$ at infinity), system (4.1) is reduced to

$$
\left\{\begin{array}{l}
\dot{z_{1}}=z_{2}, \\
\dot{z_{2}}=1 .
\end{array}\right.
$$

Obviously, system (4.2) has no equilibria. Furthermore, it has a first integral

$$
H_{1}=z_{1}-\frac{1}{2} z_{2}^{2},
$$




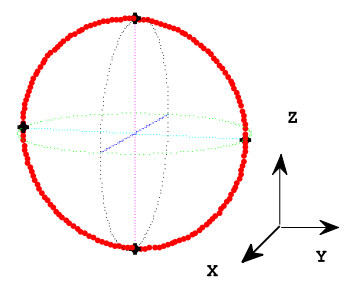

Figure 10. Phase portrait of system (1.3) at infinity.

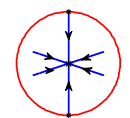

(a) $c>0$

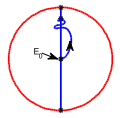

(b) $c=0$

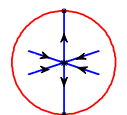

(c) $c<0$

Figure 11. Infinite heteroclinic orbits of system (1.3) for $c>0$ (left) and $c<0$ (right) when $b>0$. For $c=0$ system (1.3) has singularly degenerate heteroclinic cycles (center). The boundary of the disks corresponds to the points at infinity in the plane $y-z$, see Figure 10 .

which is shown in Figure 12.

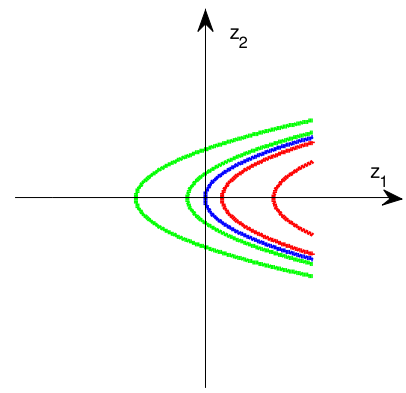

Figure 12. The phase portrait of the first integral $H_{1}=z_{1}-\frac{1}{2} z_{2}^{2}$.

The flow in the local chart $V_{1}$ is the same as the flow in the local chart $U_{1}$ reversing the time, because the compactified vector field $p(X)$ in $V_{1}$ coincides with the vector field $p(X)$ in $U_{1}$ multiplied by -1 . 


\subsubsection{In the local charts $U_{2}$ and $V_{2}$}

The expression of the Poincaré compactification $p(X)$ of system (1.3) in the local chart $U_{2}$ is as follows

$$
\left\{\begin{array}{l}
\dot{z_{1}}=(a+b) z_{1} z_{3}-a z_{3}-z_{1}^{2} z_{2}, \\
\dot{z_{2}}=(b-c) z_{2} z_{3}-z_{1} z_{2}^{2}+z_{1}^{2}, \\
\dot{z_{3}}=b z_{3}^{2}-z_{1} z_{2} z_{3} .
\end{array}\right.
$$

For $z_{3}=0$, system (4.4) turns to

$$
\left\{\begin{array}{l}
\dot{z_{1}}=-z_{1}^{2} z_{2}, \\
\dot{z_{2}}=-z_{1} z_{2}^{2}+z_{1}^{2} .
\end{array}\right.
$$

It is easy to see that system (4.4) has a line $I_{z_{2}}$ of equilibria given by the $z_{2^{-}}$ axis, and its linearized part at these equilibria has three null eigenvalues. The system (4.5) has a first integral $H_{2}=\frac{z_{2}^{2}}{z_{1}^{2}}-\frac{2}{z_{1}}$. By using the first integral one can completely describe the dynamics at infinity of system (1.3), see Figure 13.

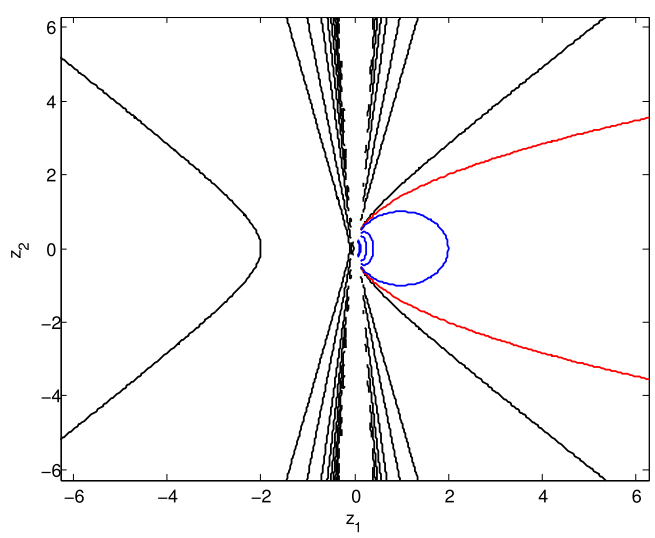

Figure 13. The phase portrait of the first integral $H_{2}=\frac{z_{2}^{2}}{z_{1}^{2}}-\frac{2}{z_{1}}$.

The flow in the local chart $V_{2}$ is the same as the flow in the local chart $U_{2}$ reversing the time, because the compactified vector field $p(X)$ in $V_{2}$ coincides with the vector field $p(X)$ in $U_{2}$ multiplied by -1 . Hence the phase portrait on the chart $V_{2}$ is obtained by reversing appropriately the time direction.

\subsubsection{In the local charts $U_{3}$ and $V_{3}$}

The expression of the Poincaré compactification $p(X)$ of system (1.3) in the local chart $U_{3}$ has the form

$$
\left\{\begin{array}{l}
\dot{z_{1}}=(a+c) z_{1} z_{3}-a z_{2} z_{3}-z_{1}^{3}, \\
\dot{z_{2}}=(c-b) z_{2} z_{3}-z_{1}^{2} z_{2}+z_{1}, \\
\dot{z_{3}}=c z_{3}^{2}-z_{1}^{2} z_{3} .
\end{array}\right.
$$


For $z_{3}=0$, system $(4.6)$ reads

$$
\left\{\begin{array}{l}
\dot{z_{1}}=-z_{1}^{3}, \\
\dot{z_{2}}=-z_{1}^{2} z_{2}+z_{1} .
\end{array}\right.
$$

Analogous to system (4.4), system (4.6) has $z_{2}$-axis as a line of equilibria $I_{z_{2}}$, too. The system (4.7) has a first integral $H_{3}=\frac{z_{2}}{\left|z_{1}\right|}-\frac{1}{2\left|z_{1}\right| z_{1}}$. The first integral may completely describe the dynamics at infinity of system (1.3), see Figure 14.

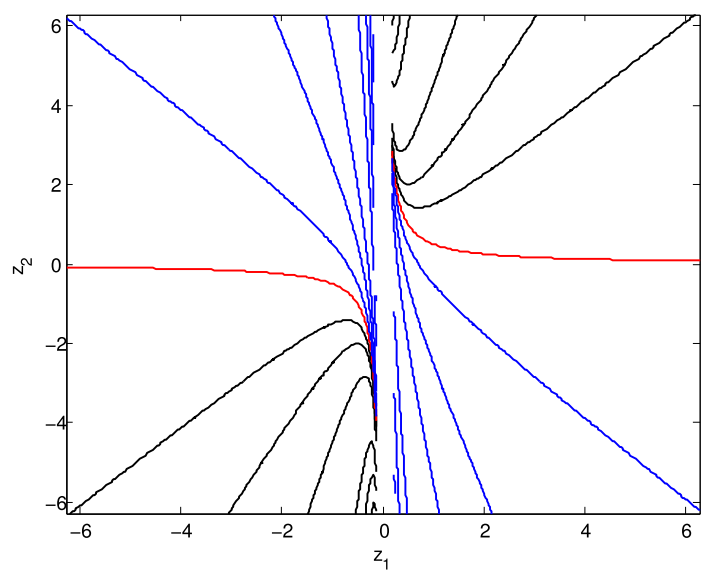

Figure 14. The phase portrait of the first integral $H_{3}=\frac{z_{2}}{\left|z_{1}\right|}-\frac{1}{2\left|z_{1}\right| z_{1}}$.

Again as in the case in Subsection 4.2.2 the flow in the local chart $V_{3}$ is the same as the flow in the local chart $U_{3}$ by reversing the time.

Now we study system (4.6) in a neighbourhood of the infinite sphere on the chart $U_{3}$ by considering $z_{3}$ small, since we are interested in the behaviour of the solutions which tends to infinity on the $z$-axis.

The $z_{3}$-axis is invariant w.r.t. the flow of system (4.6), since for $z_{1}=z_{2}=0$ system (4.6) reduces to

$$
\left\{\begin{array}{l}
\dot{z_{1}}=0, \\
\dot{z_{2}}=0, \\
\dot{z_{3}}=c z_{3}^{2} .
\end{array}\right.
$$

Hence the origin $I_{0}$ is asymptotically stable (resp. unstable) if $c<0$ (resp. $c>0$ ) along $z$-axis. Furthermore, if $c=0$, system (4.6) has a line of equilibria $I_{z_{3}}=\left(0,0, z_{3}\right)$ coinciding with the $z_{3}$-axis with corresponding eigenvalues $\lambda_{1,2}=\frac{(a-b) z_{3} \pm \sqrt{\left((a-b) z_{3}\right)^{2}+4 a b z_{3}^{2}-4 a z_{3}}}{2}$ and $\lambda_{3}=0$. Notice that $z_{3}=0$ corresponds to those points at infinity whereas $z_{3}$ sufficiently close to 0 corresponds to those points near infinity. Hence, one has the proposition as follows.

Proposition 1. Assume that $c=0$ and $z_{3}$ is sufficiently close to 0 . Then the dynamical behaviour of $I_{z_{3}}$ in the local chart $U_{3}$ is illustrated in Table 4. 
Table 4. The behaviour of $I_{3}$ in the local chart $U_{3}$.

\begin{tabular}{ll}
\hline$a-b$ & Property of $I_{3}$ \\
\hline$<0$ & a 2D $W_{l o c}^{s}$ and a $1 \mathrm{D} W_{l o c}^{c}$ \\
$=0$ & fold-Hopf bifurcation probably occurs \\
$>0$ & a 1D $W_{l o c}^{s}$, a $1 \mathrm{D} W_{l o c}^{c}$ and a $1 \mathrm{D} W_{l o c}^{u}$ \\
\hline
\end{tabular}

The flow in the local chart $V_{3}$ is the same as the flow in the local chart $U_{3}$ reversing the time. So the same type of analysis as made above, taking into account that near the infinity in the local chart $V_{3}$ we have $z_{3}$ sufficiently close to 0 , allows us to prove the following preposition.

Proposition 2. The equilibrium points $\left(0,0, z_{3}\right)$ of system (4.6) with reversed time (that is in the local chart $V_{3}$ ) are saddles normally hyperbolic to $z_{3}$-axis, that is the linear part of the system at each equilibrium $\left(0,0, z_{3}\right)$ has two real eigenvalues with opposite signs and the corresponding one-dimensional stable and unstable manifolds normal to the $z_{3}$-axis.

\subsubsection{Dynamics of system (1.3) on the sphere at infinity}

Putting together the analysis formulated in the previous subsections one has a global picture of the dynamical behaviour of system (1.3) on the sphere at infinity. It has one circle of equilibria containing the endpoints of the $y$ - and $z$-axis (see Figure 10). These are just what Theorem 6 says.

We observe that the description of the complete phase portrait of system (1.3) on the sphere at infinity is possible because of the invariance of this set under the flow of the compactified system.

\subsection{Infinite heteroclinic orbit}

For $c \neq 0$ small, $b \in \mathbb{R}$, the complex dynamical behaviour of the origin $E_{0}$ has been discussed in Section 2. The Jacobian matrix at this point has the eigenvalues $\lambda_{1}=a, \lambda_{2}=-b$ and $\lambda_{3}=-c$ with the corresponding eigenvectors $v_{1}=(1,0,0), v_{1}=(0,1,0)$ and $v_{3}=(0,0,1)$.

Hence, for $c<0$, the instability index of $E_{0}$ is at least 1 . It is easy to check that the $z$-axis is invariant under the flow of system (1.3), and it follows from the calculations above that the origin is unstable along this axis. Moreover, the equilibria at the endpoints of the $z$-axis on the sphere at infinity, which coincide with the origin $I_{0}$ in the local charts $U_{3}$ and $V_{3}$, are asymptotically stable (see, Subsection 4.2.3). Thus system (1.3) has two infinite heteroclinic orbits as in $[10,15]$, one of them consisting of the origin, the positive portion of the $z$-axis and of one equilibrium on the sphere at infinity (the endpoint of the positive $z$-axis); the other one consists of the origin, the negative part of the $z$-axis and of the endpoint of the negative $z$-axis. Particularly, one of them is shown in Figure 11(a) when $b>0$.

For $c>0$, the stability index of the saddle is at least 1 , and the origin $E_{0}$ is asymptotically stable along the $z$-axis but the endpoints of this axis on the 
sphere at infinity are unstable (see, Subsection 4.2.3). Hence, system (1.3) has also two infinite heteroclinic orbits on the $z$-axis as the ones in $[10,15]$, which are illustrated in Figure 11(c) when $b>0$.

The proof of Theorem 7 is then finished by these discussions.

\section{Conclusions}

In this paper, we introduce a conjugate Lorenz-like system. First of all, we discuss its basic local dynamical behaviors including the distribution of equilibrium points and their stabilities. Some of its other rich dynamical behaviors, such as the existence of a set of infinitely many degenerate heteroclinic cycles, the existence of homoclinic and heteroclinic orbits and the dynamics at infinity, etc., which are not studied in any known literature, are formulated in this paper.

By numerical simulations, we find that there exist chaotic attractors that are bifurcated from singularly degenerate heteroclinic cycles, homoclinic orbits to $E_{0}$, heteroclinic orbits joining $E_{0}$ and $E_{ \pm}$when its parameters do not satisfy the conditions in $[5,7]$. It is worthy to further theoretically explore the mechanism for the occurrence of such chaotic attractors in the future.

\section{Acknowledgments}

This work is partly supported by NSF of China (grant: 61473340, 10771094, 11202192, 11201360), the NSF of Yangzhou University and the Fundamental Research Funds for the Central Universities $\sharp 2652012097)$. We’d like to thank Prof. Guanrong Chen of City University of Hong Kong for his presenting us some simulation programs.

\section{References}

[1] G. Chen and T. Ueta. Yet another chaotic attractor. Int. J. Bifurcation Chaos, 9(7):1465-1466, 1999. http://dx.doi.org/10.1142/S0218127499001024.

[2] F. S. Dias, L. F. Mello and J. Zhang. Nonlinear analysis in a Lorenz-like system. Nonlinear Anal. Real World Appl., 11(5):3491-3500, 2010. http://dx.doi.org/10.1016/j.nonrwa.2009.12.010.

[3] F. Dumortier and H. Kokubu. Chaotic dynamics in $\mathbb{Z}_{2}$-equivariant unfoldings of codimension three singularities of vector fields in $\mathbb{R}^{3}$. Ergodic Theory Dynam. Systems, 20(1):85-108, 2000. http://dx.doi.org/10.1017/S0143385700000067.

[4] Z. Elhadj. Dynamical analysis of a $3-D$ chaotic system with only two quadratic nonlinearities. J. System. Sci. Complexity, 21(1):67-75, 2008. http://dx.doi.org/10.1007/s11424-008-9067-0.

[5] H. Kokubu and R. Roussarie. Existence of a singularly degenerate heteroclinic cycle in the Lorenz system and its dynamical consequences: Part I. J. Dynam. Differential Equations, 16(2):513-557, 2004. http://dx.doi.org/10.1007/s10884-004-4290-4.

[6] Y. A. Kuzenetsov. Elements of Applied Bifurcation Theory, third ed. SpringerVerlag, New York, 2004. 
[7] X. Li and Q. Ou. Dynamical properties and simulation of a new Lorenz-like chaotic system. Nonlinear Dynam., 65(3):255-270, 2011. http://dx.doi.org/10.1007/s11071-010-9887-z.

[8] X. Li and H. Wang. Homoclinic and heteroclinic orbits and bifurcations of a new Lorenz-type system. Int. J. Bifurcation Chaos, 21(9):2695-2712, 2011. http://dx.doi.org/10.1142/S0218127411030039.

[9] X. Li and P. Wang. Hopf bifurcation and heteroclinic orbit in a 3D autonomous chaotic system. Nonlinear Dynam., 73(1-2):621-632, 2013.

[10] Y. Liu. Dynamics at infinity and the existence of singularly degenerate heteroclinic cycles in the conjugate Lorenz-type system. Nonlinear Anal. Real World Appl., 13(6):2466-2475, 2012. http://dx.doi.org/10.1016/j.nonrwa.2012.02.011.

[11] Y. Liu, S. Pang and D. Chen. An unusual chaotic system and its control. Math. Comput. Model, 57(9):2473-2493, 2013. http://dx.doi.org/10.1016/j.mcm.2012.12.006.

[12] E. N. Lorenz. Deterministic nonperiodic flow. J. Atmos. Sci., 20(2):130-141, 1963. http://dx.doi.org/10.1175/1520-0469(1963)020ز0130:DNF ¿2.0.CO;2.

[13] J. Lü and G. Chen. A new chaotic attractor coined. Int. J. Bifurcation Chaos, 12(3):659-661, 2002. http://dx.doi.org/10.1142/S0218127402004620.

[14] T. Matsumoto, M. Komuro, H. Kokubu and R. Tokunaga. Bifurcation - Sights, Sounds and Mathematics. Springer-Verlag, New York, 1993.

[15] M. Messias. Dynamics at infinity and the existence of singularly degenerate heteroclinic cycles in the Lorenz system. J. Phys. A, 42(11):115101 (18 pp.), 2009. http://dx.doi.org/10.1088/1751-8113/42/11/115101.

[16] O. E. Rössler. An equation for continuous chaos. Phys. Lett. A, 57(5):397-398, 1976. http://dx.doi.org/10.1016/0375-9601(76)90101-8.

[17] Z. Wei and Q. Yang. Dynamics analysis of a new autonomous 3-D chaotic system only with stable equilibria. Nonlinear Anal. Real World Appl., 12(1):106-118, 2011. http://dx.doi.org/10.1016/j.nonrwa.2010.05.038.

[18] X. Xiong and J. Wang. Conjugate Lorenz-type chaotic attractors. Chaos Solitons Fractals, 40(2):923-929, 2009. http://dx.doi.org/10.1016/j.chaos.2007.08.087.

[19] Q. Yang and G. Chen. A unified Lorenz-type system and its canical form. Int. J. Bifurcation Chaos, 16(10):2855-2871, 2006. http://dx.doi.org/10.1142/S0218127406016501.

[20] Q. Yang, Z. Wei and G. Chen. An unusual 3D autonomous quadratic chaotic system with two stable node-foci. Int. J. Bifurcation Chaos, 20(4):1061-1083, 2010. http://dx.doi.org/10.1142/S0218127410026320. 